\title{
A Study of $\mathrm{Mn}^{2+}$ Doping in CdS Nanocrystals
}

Angshuman Nag, ${ }^{1}$ Sameer Sapra, ${ }^{1}$ C. Nagamani, ${ }^{1}$ Ajay Sharma,${ }^{2}$ N. Pradhan, ${ }^{3}$ S. V. Bhat ${ }^{2}$ and D. D. Sarma ${ }^{1,3, *}$

\section{Supporting Information Available}

1 Solid State and Structural Chemistry Unit, Indian Institute of Science, Bangalore-560 012, India

2 Department of Physics, Indian Institute of Science, Bangalore-560 012, India

${ }^{3}$ Centre for Advanced Materials, Indian Association for the Cultivation of Science, Kolkata700032, India 
Figure 1: X-ray diffraction pattern of $\mathrm{MnS}$ synthesized following the same procedure of synthesizing $25 \%$ nominal Mn doped CdS nanocrystals (NCs), but without adding the $\mathrm{Cd}^{2+}$ precursor.

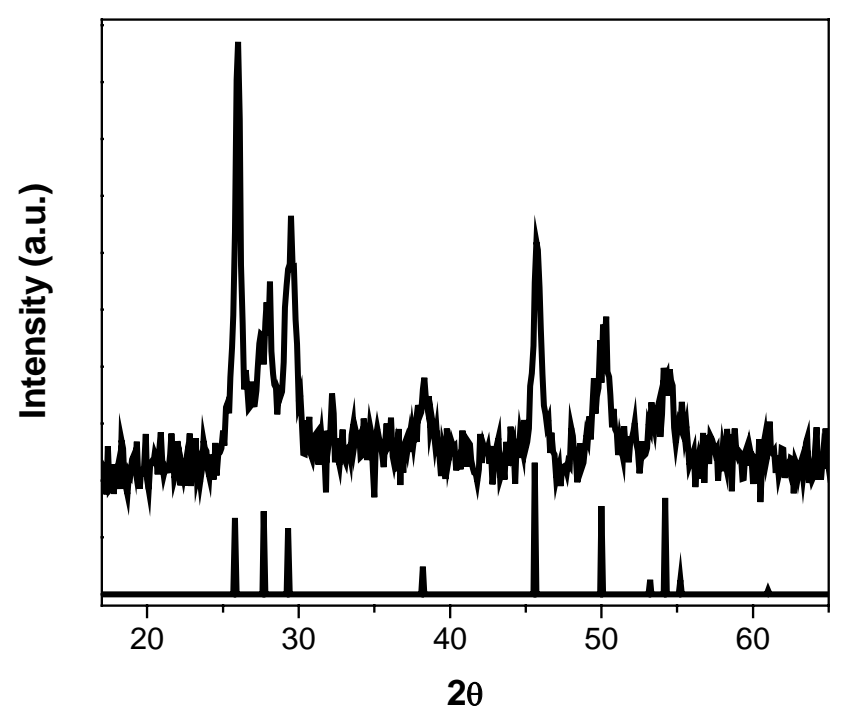

Figure 2: Photoluminescence (PL) spectra of 1.9\% Mn doped CdS NCs with different time delay.

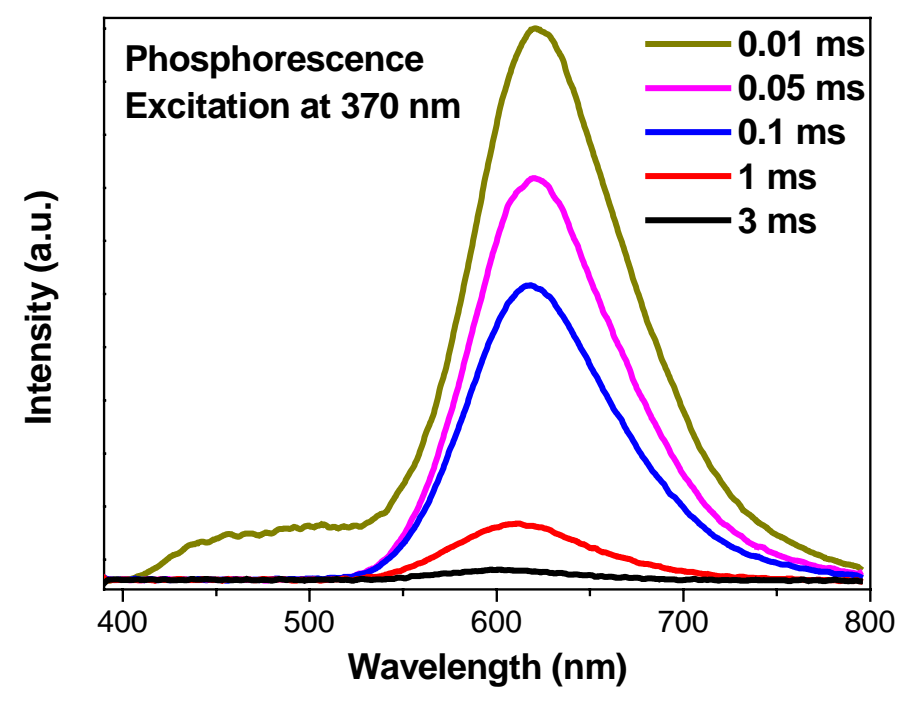


Figure 3: PLE spectra of the undoped and 1.9\% doped NCs corresponding to 514 and $620 \mathrm{~nm}$ emission, respectively.

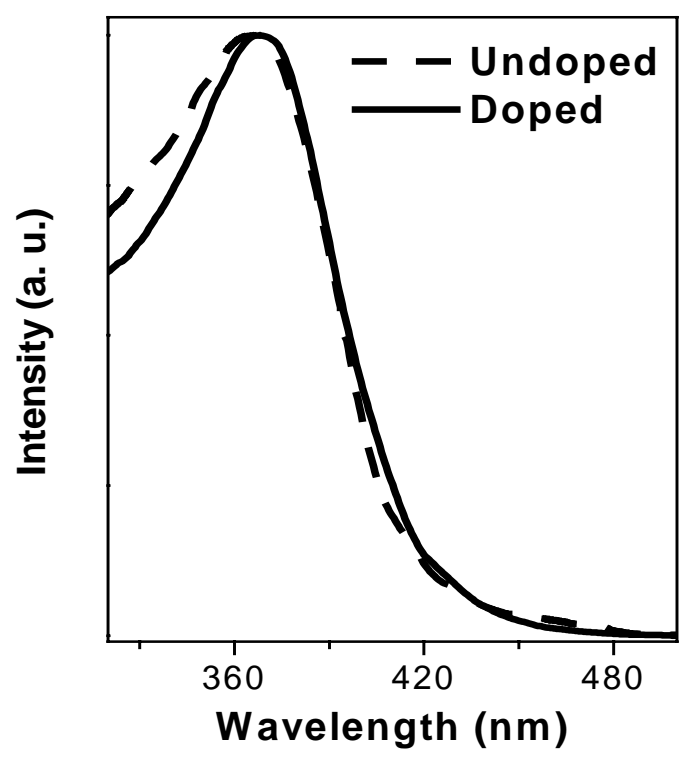

Figure 4: PL emission spectra normalized by the corresponding absorption spectra for different synthesis temperature.

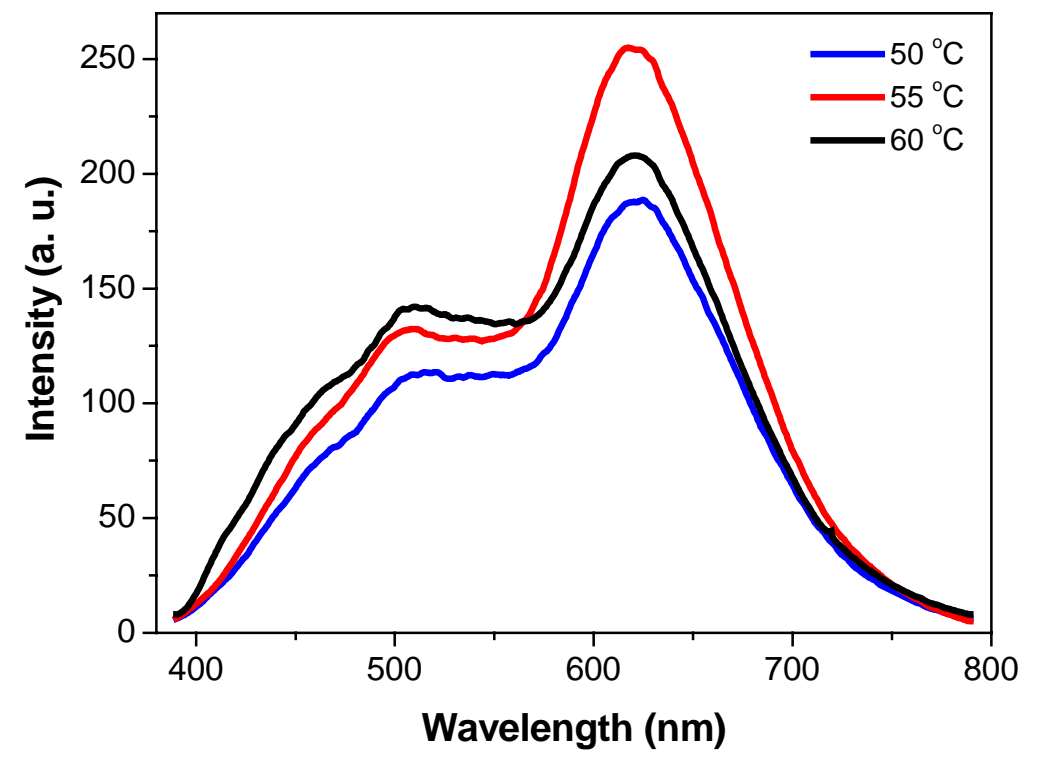


Figure 5: PL spectra of 1.9\% Mn doped CdS nanocrystals at different excitation wavelength.

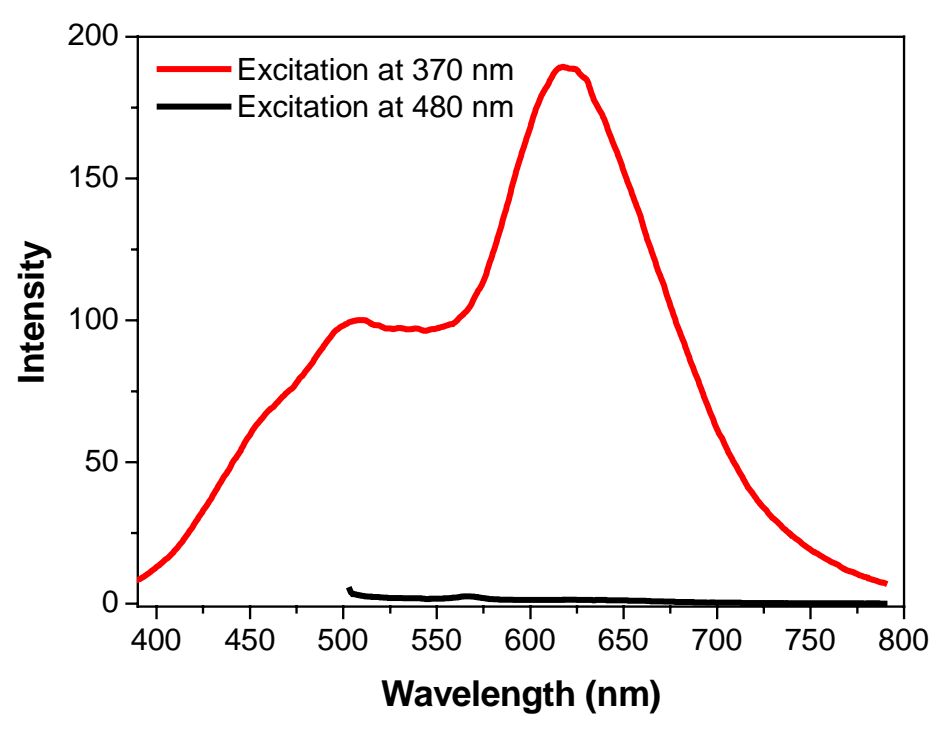

Figure 6: Photographs of the powders of larger and smaller fractions of the $1.9 \% \mathrm{Mn}$ doped CdS nanocrystals after size selective precipitation.

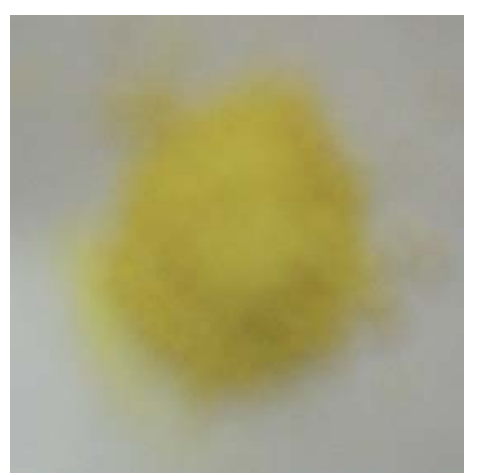

Larger particles

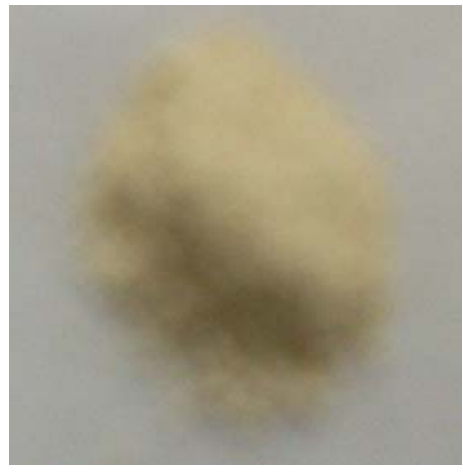

Smaller particles 


\section{$\mathrm{Mn}^{2+} \mathrm{d}$ emission well separated from surface state emissions:}

One important difference appear to be that surface state emissions with peak at $\sim 508 \mathrm{~nm}$ exhibited by these NCs are much more blue shifted compared to earlier reports; this in turn allows us to observe distinct PL signature for the $\mathrm{Mn}^{2+} d$-emission (peak at $\sim 620 \mathrm{~nm}$ ) well separated from surface state emissions. Nature of surface state emissions depends mainly on the capping agent, particle size, reaction temperature and surface stoichiometry of the NCs. In general, it is difficult to tune the energies arising from such surface states, rendering it difficult to influence the emission by rational synthesis. However, a red shift in surface state emission has been observed with the increase in particle size. ${ }^{1}$ Particle size $(\sim 2 \mathrm{~nm})$ achieved by us is much smaller in size compared to most of previous reports, suggesting one plausible reason for the observed blue shift of surface emission. But this cannot be the only reason, since there are a few reports on similar sized CdS particles with overlapping surface state emissions and $\mathrm{Mn}^{2+} d$ emission. The unique feature of our synthesis compared to earlier reports on $\mathrm{Mn}^{2+}$-doped CdS NCs is the use of 1-thioglycerol as a capping agent. A careful investigation of literature reveals a few reports ${ }^{2,3}$ of undoped CdS NCs of $\sim 2$ nm diameter synthesized using similar capping agents, which exhibit surface state emissions similar to our results with maximum in the range of 480$500 \mathrm{~nm}$; unfortunately $\mathrm{Mn}^{2+}$-doping was not carried out in any of these cases. Under the achieved size limits in our synthesis, we believe 1-thioglycerol is playing an important role to keep the surface state emissions blue shifted compared to the earlier reports of $\mathrm{Mn}^{2+}$-doped CdS NCs and eventually separating out surface state emissions and $\mathrm{Mn}^{2+} d$-emission.

\section{References:}

1) Rao, C. N. R.; Muller, A. k.; Cheetham, A. K. The Chemistry of Nanomaterials, Wiley- VCH,

Chapter 11, vol. 2. 
2) Artemyev, M. V.; Sperling, V.; Woggona, U J. Appl. Phys. 1997, 81, 6975

3) Torimoto, T.; Kontani, H.; Shibutani, Y.; Kuwabata, T. S.; Mori, H.; Yoneyama, H. J. Phys. Chem. B 2001, 105, 6838 